\title{
PERCEPÇÃO: UMA CAIXA PRETA PARA MARKETING?
}

\author{
PERCEPTION: A BLACK BOX TO MARKETING?
}

Recebido em 14.07.10 / Aceito em 05.12.10

\section{Braulio Oliveira, ${ }^{1}$ Gracieli Regina Mendes Tavares ${ }^{2}$ e Karla Satiko Sato ${ }^{3}$}

\begin{abstract}
Resumo
Um dos elementos menos estudado pela literatura de marketing, mas que é de fundamental importância para a compreensão do processo de decisão de compra, é a percepção. Pensar em percepção não é somente pensar em como um consumidor enxerga as suas opções de compra, mas também entender os mecanismos envolvidos para esse sentido. O presente artigo tem como objetivo discutir a percepção humana sob a perspectiva de marketing. Trata-se de um trabalho de cunho teórico, com objetivos exploratórios, baseado na revisão da literatura relativa ao assunto em questão. Os estímulos são percebidos pelo indivíduo por meio de suas capacidades, ou seja, por cada pessoa em suas individualidades mentais e perceptivas. Ficou evidenciada a forte relação existente entre a percepção e os estímulos sensoriais. Desvendar o funcionamento da formação da percepção pode resultar na compreensão das ações dos consumidores que, atualmente, são consideradas emocionais e que fogem das previsibilidades das empresas. Estudar a percepção traz a possibilidade de entender como a informação chega ao indivíduo e se torna consciente. As limitações do trabalho dizem respeito ao fato e se tratar de um ensaio, ficando para estudos futuros o envolvimento de pesquisas empíricas compatíveis com as problemáticas que forem trabalhadas envolvendo a percepção.
\end{abstract}

Palavras-chave: Marketing. Percepção. Comportamento do consumidor.

1 Doutor em Administração pela FEA/USP. Professor do Departamento de Administração da FEI/SP. Email: braulio@fei.edu.br

2 Mestranda em Administração na FEI/SP. Email: gracielitavares@yahoo.com.br

3 Mestranda em Administração na FEI/SP. Email: karlasatikosato@msn.com 


\begin{abstract}
One of the least studied elements in the marketing literature, but which is of fundamental importance for understanding the process of buying decision is perception. Thinking of perception is not only thinking about how a consumer sees their options, but also to understand the mechanisms involved in this direction. This article aims to discuss human perception from the perspective of marketing. This is a work of theoretical, with exploration goals, based on review of the literature on the subject. The stimuli are perceived by the individual through its capabilities, ie, for each individual in their personalities, mental and perceptive. It was evident the strong relationship between perception and sensory stimuli. Unravel the workings of the formation of perception can result in understanding the actions of consumers that are currently considered emotional, and fleeing the predictability of business. Studying the perception brings the ability to understand how information reaches the person and becomes conscious. The limitations of the study concern the fact and it is a test, leaving for future studies involving empirical research consistent with the issues that are worked involving perception.
\end{abstract}

Keywords: Marketing. Perception. Consumer behavior.

\title{
1 INTRODUÇÃO
}

Devido à sua própria identidade e à competitividade, as realizações de marketing dependem de conhecer e entender o consumidor - tanto os fatores que o influenciam, quanto o processo de decisão de compra (HAWKINS; MOTHERSBAUGH; BEST, 2007). Mas o desafio é grande, pois as pessoas são diferentes entre si por fatores diversos, como cultura, classe social e psicologia. (BLACKWELL; MINIARD; ENGEL, 2005).

Um dos elementos menos estudado pela literatura de marketing, mas que é de fundamental importância para a compreensão do processo de decisão de compra, é a percepção.

Pensar em percepção não é somente pensar em como um consumidor enxerga as suas opções de compra, mas também entender os mecanismos envolvidos para esse sentido. Conhecer o indivíduo, assim como entender as escolhas feitas por ele ao adquirir um produto ou serviço vincula-se ao conhecimento da percepção humana. Afinal, "as pessoas percebem os produtos e seus atributos de acordo com suas próprias expectativas; portanto, seu processo decisório tende a ir ao encontro dos elementos do ambiente que são mais importantes para elas". (GUILHOTO, 2001, p. 57).

Segundo Hawkins, Mothersbaugh e Best (2007, p. 114), a percepção "é um processo que começa com a exposição e a atenção do consumidor aos estímulos de marketing e termina com a interpretação do consumidor". Os autores ressaltam a importância do entendimento da percepção dos consumidores para a área de marketing, pois a eficácia na transmissão de mensagens aos públicos-alvo depende dessa compreensão, além de outros fatores. A percepção está presente nos três primeiros estágios do processamento de informação para a tomada de decisão - exposição, atenção e interpretação -. Os dois primeiros são altamente seletivos, e o último é extremamente subjetivo, conforme apresenta-se na figura 1.

Para Guilhoto (2001), a percepção é importante para o estudo do comportamento do consumidor, pois os consumidores são movidos e motivados a comprar pelas percepções que tem dos produtos, marcas e empresas, já que, raramente, conseguem adquirir informações totais.

Rev. Adm. UFSM, Santa Maria, v. 3, n. 3, p. 424-430, set./dez. 2010 
Machado e Damacena (2006) afirmam que desvendar os mistérios dos processos de interpretação, cognição e comportamento do consumidor é uma tarefa complexa e inesgotável, e que estudos desses processos são fundamentais para marketing, para proporcionar ganhos tanto organizacionais, quanto para seus consumidores.

A filosofia também se coloca a estudar a percepção e a discuti-la sob o ponto de vista de diferentes teorias. Searle (1995) coloca o conhecimento da percepção de modo a entendê-la como um dos mecanismos mentais facilitadores entre o meio e a mente. O filósofo coloca em questão a percepção como forma de intencionalidade.

Durozoi e Roussel (1993) interligam, nos estudos filosóficos, o conhecimento de percepção aos trabalhos de Memória em Bérgson e de Experiência com Kant. E, para a psicanálise, segundo Laplanche e Pontalis (1996), a consciência remete-se à percepção do mundo exterior através das possibilidades sensoriais.

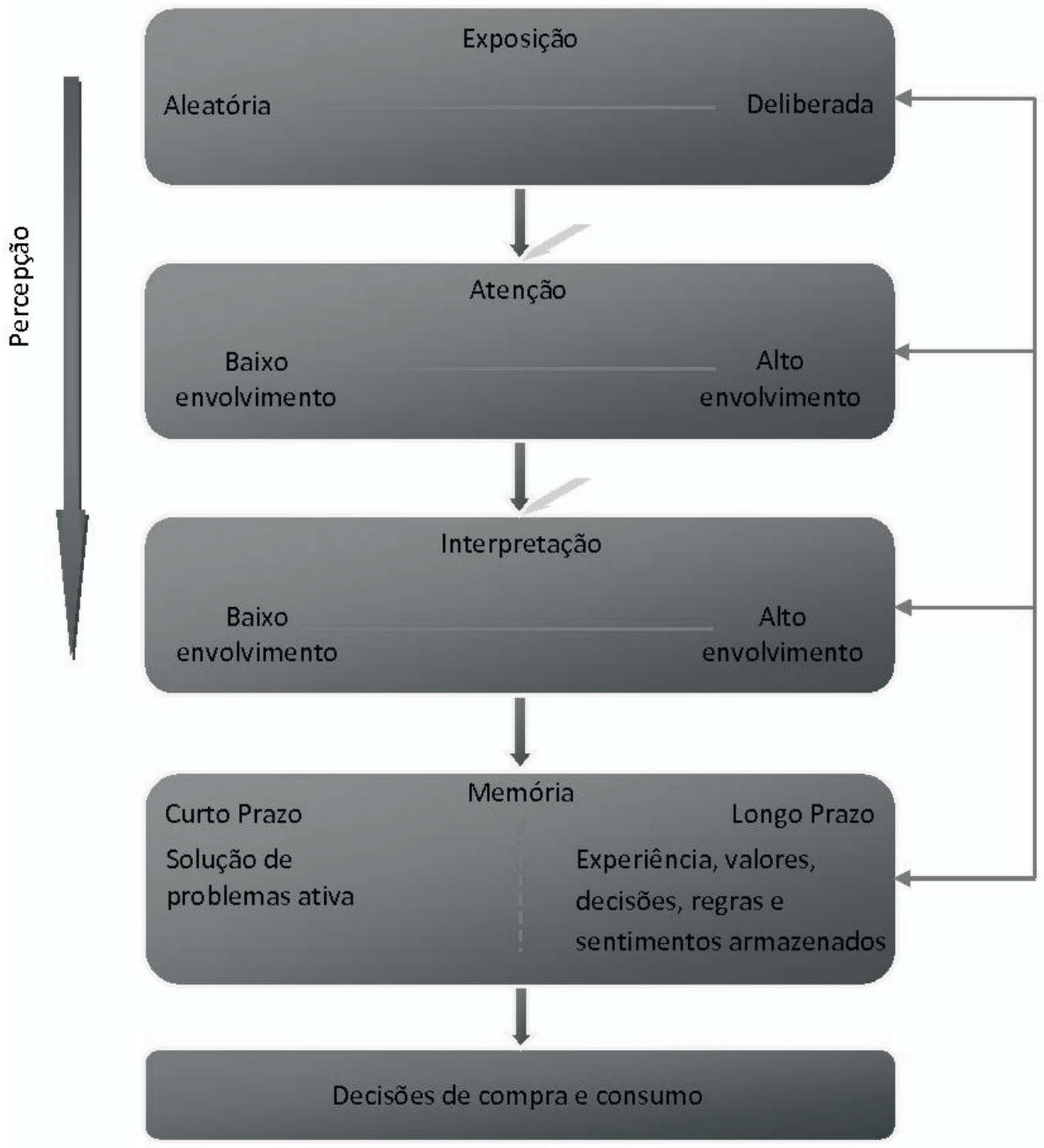

Figura 1 - Processamento de informação para a tomada de decisão do consumidor Fonte: Hawkins, Mothersbaugh e Best (2007, p. 114). 
Ao inserir o tema percepção é comum que o debate seja discorrido a partir da utilização do campo da visão como meio perceptivo, mas é preciso pensar que os outros sentidos também contribuem e participam nesse processo.

O perceber de cada pessoa está associado a uma série de outras capacidades mentais, ligadas à aprendizagem, memória, atenção além da pré-disposição. A "intencionalidade", citada por Searle proporciona ao indivíduo a percepção. E esta é realizada de acordo com as demais possibilidades acima citadas.

Em vista da escassez de trabalhos da área de marketing que abordam deliberadamente a percepção, este artigo tem por principal objetivo discutir o assunto percepção com foco de marketing.

Assim, trata-se de um trabalho de cunho teórico, com objetivos exploratórios, baseado na revisão da literatura relativa ao assunto em questão. Foram utilizados manuais, livros e artigos acadêmicos de marketing e de outras áreas de conhecimento, como filosofia, psicologia e psicanálise, de âmbito nacional e internacional.

\section{COMPORTAMENTO DO CONSUMIDOR}

As empresas direcionadas para o mercado buscam pesquisar e entender os seus públicos-alvo, pois essas informações são fundamentais para a tomada de decisão de marketing. (HOOLEY; SAUNDERS; PIERCY, 2006).

Segundo Solomon (2002, p. 24), o comportamento do consumidor é "o estudo dos processos envolvidos quando indivíduos ou grupos selecionam, compram, usam ou dispõem de produtos, serviços, idéias ou experiências para satisfazer necessidades e desejos".

Hawkins, Mothersbaugh e Best (2007, p. 4) ampliam a definição de Solomon ao definirem o comportamento do consumidor como o

estudo de indivíduos, grupos ou organizações e o processo que eles usam para selecionar, obter, usar e dispor de produtos, serviços, experiências ou idéias para satisfazer necessidades e o impacto que esses processos têm sobre o consumidor e a sociedade.

Nesta visão, o campo de pesquisa do comportamento do consumidor torna-se fundamental para o entendimento de vários fatores do processo, ou seja, o comportamento do consumidor é considerado como um processo cíclico envolvendo influências antes, durante e após a compra. (SOLOMON, 2002).

Conforme se pode observar na figura 2, a percepção é a primeira etapa do conjunto de influências internas do processo de decisão de compra do consumidor.

\section{PERCEPÇÃO}

Quando se fala em percepção, é possível descobrir significados variados sobre o assunto, tais como na maneira como um objeto é visto, do conceito que se faz sobre algum produto ou pessoa, da lembrança que existe de algum momento.

De fato, a percepção pode ser vinculada a todos esses sentidos. Para fins dos estudos focados em marketing, um conceito apropriado é o de Paim (1993, p. 9): "ato pelo qual toma- 


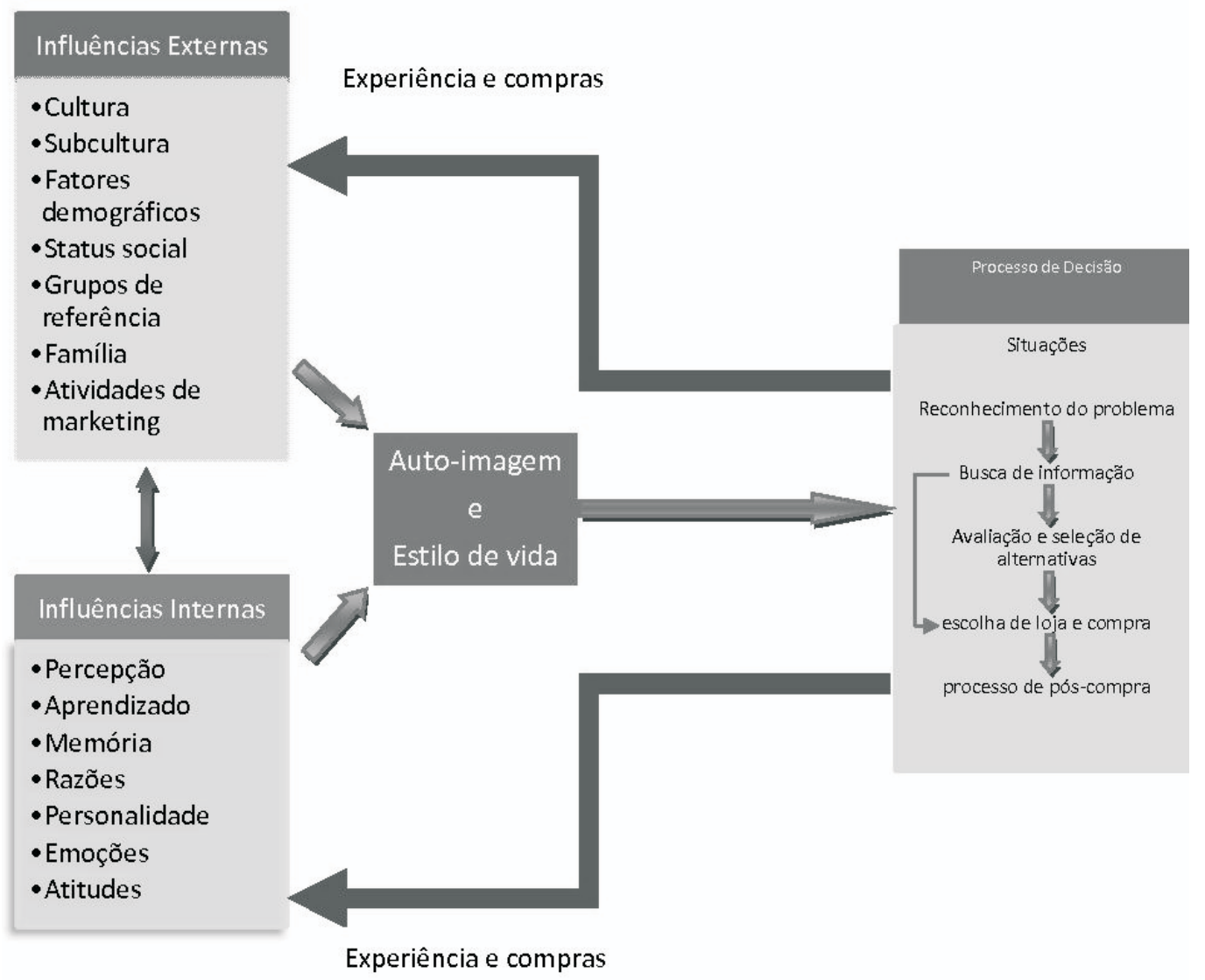

Figura 2 - Modelo de comportamento do consumidor

Fonte: Hawkins, Mothersbaugh e Best (2007, p. 19).

mos conhecimento de um objeto do meio exterior, considerado como real, isto é, existente fora da própria atividade perceptiva."

O desenvolvimento de percepção depende de que objetos, cores e movimentos atinjam um dos quatro sentidos humanos. Engloba sensações, expectativas e experiências do indivíduo.

As sensações - resultantes de uma decodificação em sinais neurais de sistemas do ambiente, como exemplo iluminação, temperatura e sons - é fundamental para a formação da percepção, pois fazem parte dela, após serem selecionadas, organizadas e interpretadas. (LERSCH, 1966).

Myers (2003) considera que, no dia a dia, as sensações, experiências e expectativas individuais são interpretadas pela mente. O autor define como bottom-up as sensações que chegam à mente e top-down às expectativas e experiências do indivíduo.

De acordo com Vigotski (2008), a percepção muda de acordo com o crescimento do indivíduo, desde a infância.

Para Bee (1986), a percepção está ligada a "saber", a "prestar atenção". Assim, com o desenvolvimento perceptivo, a criança passa a aprender o que pode ignorar e para onde deve dirigir sua atenção.

Vigotski (2008) insere o conhecimento da alteração da percepção com o início da fala no desenvolvimento infantil. Para ele, aos poucos a aprendizagem da fala possibilita à criança mudanças em sua atividade perceptiva. A visão, principal canal desta atividade até a aprendizagem linguística, passa a ser vinculada também à percepção verbalizada. Com seu amadurecimento, a 
criança deixa aos poucos de isolar um único objeto e já é capaz de verbalizar sobre outros, o que o autor chama de "Percepção Verbalizada".

Ao começar a falar, pode-se perceber que a criança separa um objeto de um contexto e o rotula. Conforme vai ampliando seu conhecimento em palavras e amadurecendo o processo cognitivo, a percepção infantil se torna aos poucos menos imediata e mais "planejada". VIGOTSKI, 2008).

A percepção, segundo Vigotsky (2008), tem total relação com os movimentos, que, na criança, são lentos e diversos. A criança pode fazer sua escolha e refazê-la, enquanto o adulto faz a escolha a partir de um movimento já realizado anteriormente.

A percepção então se altera no decorrer da vida. Seu conhecimento intelectual, suas experiências e expectativas alteram-se assim como suas motivações. O indivíduo não tem consciência de suas totais mudanças e também não conhece as alterações na sua percepção.

Daí depreende-se que o consumidor é alguém capaz de referenciar suas sensações expectativas e experiências em torno de um desejo.

Krech e Crutchfield (1980) sinalizam a importância de considerar diferenças culturais e os valores por elas sustentadas na formação da percepção de um indivíduo.

No sentido de escolhas, Schiffman e Kanuk (2000) definem "Seleção Perceptiva" como os estímulos que serão considerados na formação da percepção, em meio a estímulos diversos.

Krech e Crutchfield (1980) trazem o conhecimento de que a percepção de um indivíduo pode ser diferente de acordo com sua "relação" com o objeto. Conhecer um determinado objeto, segundo os autores, diferencia a maneira dele ser percebido, assim como a motivação também pode ser fator de alteração da percepção. Para esses autores, também a motivação e a personalidade do indivíduo tem papel importante no processo de percepção, a atração ou repulsa a um objeto tem total ligação ao comportamento (KRECH; CRUTCHFIELD, 1980).

Sheth, Mittal e Newman (2001) acreditam que as referências e crenças pessoais podem causar o que chamam de "distorção perceptual" - uma maneira bastante individual de decodificar o que está sendo transmitido.

Doenças físicas e mentais ou mesmo alterações hormonais podem interferir na percepção (KRECH; CRUTCHFIELD, 1980). Myers (2003) lembra que existem possibilidades de falhas entre as sensações e o processo perceptivo.

A percepção inicia-se a partir do contato do indivíduo com o meio, o que se dá através da interação com os estímulos sensoriais. O sistema nervoso central recebe, através dos receptores sensoriais, uma série de sensações constantemente e os neurônios são estimulados em regiões diferentes do cérebro. (CARTER, 2003).

\section{CONSIDERAÇÕES FINAIS}

Avançar no estudo do comportamento do consumidor é fundamental para que as empresas possam trabalhar a filosofia de marketing em sua plenitude.

Ainda que o uso de tecnologia de informação contribua para a compreensão do consumidor, algumas lacunas permanecem. Uma delas é a formação da percepção.

Os estímulos são percebidos pelo indivíduo por meio de suas capacidades, ou seja, por cada pessoa em suas individualidades mentais e perceptivas.

Fica evidenciada a forte relação existente entre a percepção e os estímulos sensoriais (e a forma como cada indivíduo o interpreta). Como no contexto de marketing, o consumidor pode receber uma série de estímulos, o "contraste" é de grande importância para que seja notado, e 
não simplesmente filtrado (SCHIFFMAN; KANUK, 2000). Mas é fato que o indivíduo irá prestar atenção maior a algo que esteja relacionado com suas necessidades e desejos. Da mesma forma que a defesa perceptiva irá afastar do indivíduo informações que possam, para este, ser desagradáveis. Além disso, de acordo com a "Lei de Weber", quanto mais intenso for o estímulo original, maior deverá ser a mudança para que seja notada. (SOLOMON, p. 58).

Desvendar o funcionamento da formação da percepção pode resultar na compreensão das ações dos consumidores que atualmente são consideradas emocionais e que fogem das previsibilidades das empresas. Estudar a percepção traz a possibilidade de entender como a informação chega ao indivíduo e se torna consciente.

Assim, evidencia-se um vasto campo de pesquisa a ser explorado, inclusive em âmbito multidisciplinar, dada a magnitude e a complexidade das questões relacionadas à mente humana.

\section{REFERÊNCIAS BIBLIOGRÁFICAS}

BEE, H. A criança em desenvolvimento. São Paulo: Harbra, 1986.

BLACKWELL, Roger D.; MINIARD, Paul W.; ENGEL, James F. Comportamento do consumidor. São Paulo: Pioneira Thomson Learning, 2005.

CARTER, R. O livro de ouro da mente. Rio de Janeiro: Ediouro, 2003.

DUROZOI, G; ROUSSEL, A. Dicionário de filosofia. São Paulo: Papirus, 1993.

GUILHOTO, L. de F. M. A influência do país de origem na percepção do consumidor sobre a qualidade dos produtos. Caderno de Pesquisas em Administração, v. 8, n. 4, p. 53-64, out./dez. 2001.

HAWKINS, D. I.; MOTHERSBAUGH, D. L.; BEST, R. J. Comportamento do consumidor: construindo a estratégia de marketing. Rio de Janeiro: Elsevier, 2007.

HOOLEY, Graham J.; SAUNDERS, John A.; PIERCY, Nigel F. Estratégia de marketing e posicionamento competitivo. São Paulo: Pearson, 2006.

KRECH, D.; CRUTCHFIELD, R. Elementos de psicologia. São Paulo: Pioneira, 1980.

LAPLANCHE, J.; PONTALIS, J. B. Vocabulário da psicanálise. São Paulo: Martins Fontes, 1996.

LERSCH, P. La estrutura de la personalidad. Barcelona: Scientia, 1966.
MACHADO, S. K.; DAMACENA, C. Percepção dos consumidores acerca do marketing relacionado a causas: uma revisão da literatura. BASE - Revista de Administração e Contabilidade da Unisinos, v. 3, n. 2, p. 93-101, maio/ago. 2006.

MYERS, D. G. Explorando a psicologia. Rio de Janeiro: LTC, 2003.

PAIM, I. Curso de psicolpatologia. São Paulo: EPU, 1993.

SCHIFFMAN, L. G.; KANUK, L. L. Comportamento do consumidor. Rio de Janeiro: LTC, 2000.

SEARLE, J. R. Intencionalidade. São Paulo: Martins Fontes, 1995.

SHETH, J. N.; MITTAL, B.; NEWMAN, B. I. Comportamento do cliente: indo além do comportamento do consumidor. São Paulo: Atlas, 2001.

SOLOMON, M. R. O comportamento do consumidor: comprando, possuindo e sendo. Porto Alegre: Bookman, 2002.

VIGOTSKI, L. S. A formação social da mente. São Paulo: Martins Fontes, 2008. 